Portland State University

PDXScholar

1978

\title{
An instrument for determination of the polarization of fluorescence
}

George Irwin Johnston

Portland State University

Follow this and additional works at: https://pdxscholar.library.pdx.edu/open_access_etds

Part of the Chemical Engineering Commons

Let us know how access to this document benefits you.

\section{Recommended Citation}

Johnston, George Irwin, "An instrument for determination of the polarization of fluorescence" (1978). Dissertations and Theses. Paper 2800.

https://doi.org/10.15760/etd.2796

This Thesis is brought to you for free and open access. It has been accepted for inclusion in Dissertations and Theses by an authorized administrator of PDXScholar. Please contact us if we can make this document more accessible: pdxscholar@pdx.edu. 
AN ABSTRACT OF THE THESIS OF George Irwin Johnston for the Master of Science in Applied Science presented December 12, 1978.

Title: An Instrument for Determination of the Polarization of Fluorescence.

APPROVED BY. MEMBERS OF THE THESIS COMMITTEE:

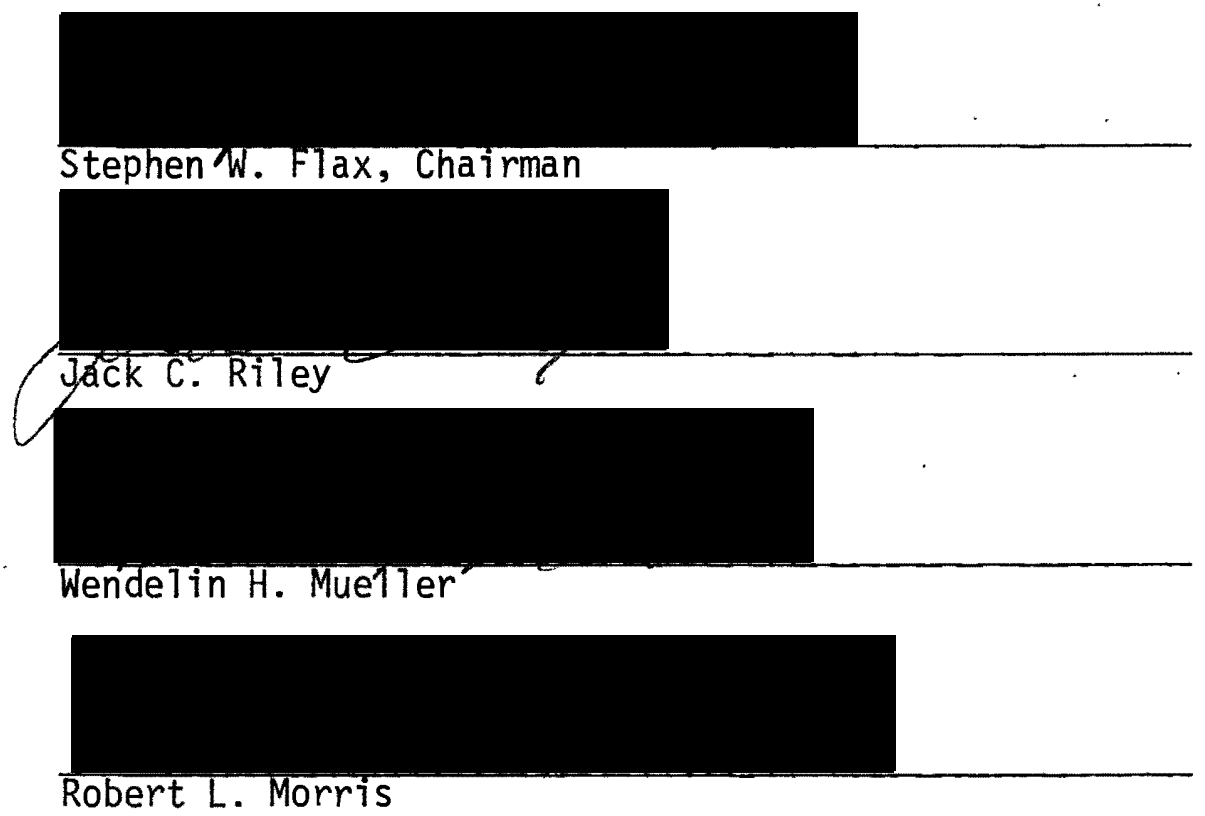

This thesis is concerned with the development of a compact, sensitive and reliable instrument for determination of the polarization of fluorescence of fluorescein tagged molecules in solution. The instrument as designed and constructed differs from others $(2,3,4,5,6,8,9)$. The difference is that all of its optical system is aligned on a single axis. This was accomplished by the use of an ellipsoidal mirror positioned between the excitation source and the phototube fluorescence 
signal receiver. In addition, the excitation source, a 45 watt tungsten-iodide light was electronically regulated to control the intensity.

Two types of determinations were made to verify the performance of the instrument. The first was the absolute sensitivity to varying fluorescein concentrations. Concentrations of fluorescein in 0.1 molar sodium phosphate buffer were prepared ranging from $10^{-6}$ molar to $10^{-11}$ molar. After the minimum detectable concentration was determined, five runs on samples of each concentration from the minimum detectable to $10^{-6}$ were made. The second check was for the sensitivity and linearity of the system in the measurement of polarization. Solutions of glycerol ranging from $62 \%$ to $90 \%$ were prepared with $10^{-6}$ molar fluorescein concentration.

The data from the fluorescein sensitivity and polarization runs were averaged and the averages plotted on linear paper. In each case the instrument exhibited excellent linear response and reasonable standard deviation. 


\title{
AN INSTRUMENT FOR DETERMINATION \\ OF THE POLARIZATION OF FLUORESCENCE
}

by

GEORGE IRWIN JOHNSTON

A thesis submitted in partial fulfillment of the requirements for the degree of

\author{
MASTER OF SCIENCE \\ in \\ APPLIED SCIENCE
}

Portland State University

1979 
TO THE OFFICE OF GRADUATE STUDIES AND RESEARCH:

The members of the Committee approve the thesis of George Irwin Johnston presented December 12, 1978.

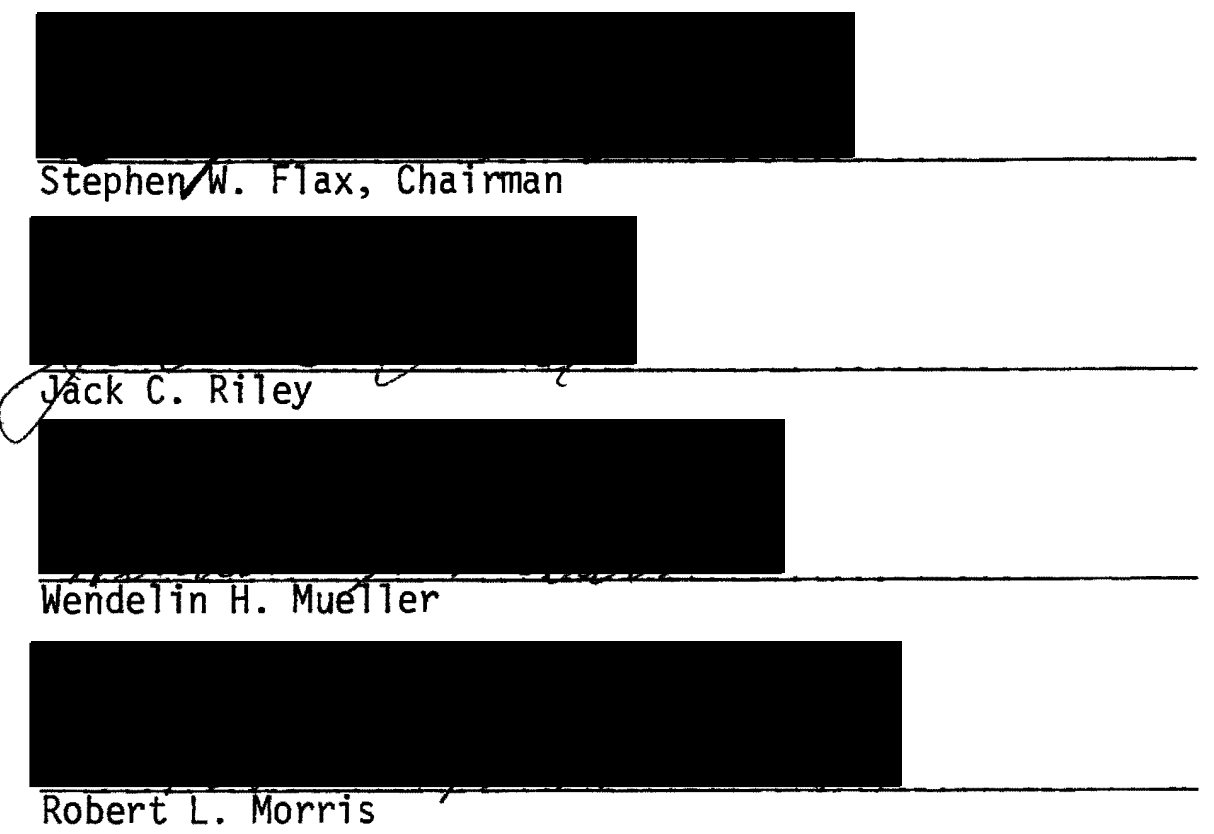

APPROVED:

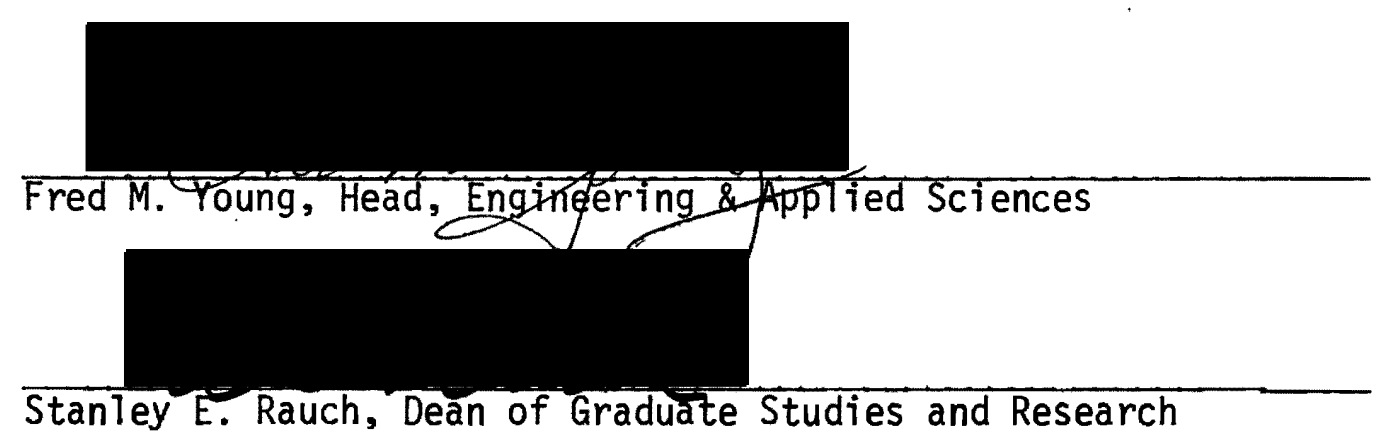




\section{ACKNOWLEDGEMENTS}

No one does a project of this type and magnitude without outside help. And I had so much of it that it is hard to know where to start giving credit and thanks.

There was Steve Flax of the PSU Engineering and Applied Science Department who encouraged me into graduate school and initially assisted me in getting started on my thesis project. He felt it was a useful and challenging project and $I$ believe his judgement has been proven right.

Then there was Bob Morris and Larry Bowers of the University of Oregon Health Sciences Center, Clinical Pathology Department. They had long been interested in the development of such an instrument. In return they were my consultants in the physics and chemistry of the system.

During the construction phase I received much help from members of my department at the Health Sciences Center, Instrument \& Safety Services. Ray 01iver and Jim McVein were of enormous help in the mechanical construction of the system and the later debugging. Dick Crumley and Severo DeGuzman were of equal assistance in the electronic development. Then during the testing phase my Safety Officer, Jim Joyce, who happens to possess a chemistry background became of incalculable value. It was he who made up the required solutions and spent many hours assisting me with the data runs. 
Then there was Jack Riley, also of the PSU Engineering and Applied Science Department, who came to my rescue when I had difficulty sorting out some of the theory of polarization.

Finally, and certainly not least, there is the person who helps you communicate all this information to the outside world. The excellent typing of the manuscript was performed by my office manager, Jeanne Bunch.

To all of you may I say "Many Thanks" for making this a wonderful and rewarding year. 
TABLE OF CONTENTS

PAGE

ACKNOWLEDGEMENTS ..................... $i i_{i}$

LIST OF FIGURES .................. . . vi

INTRODUCTION AND HISTORY ................. 1

THEORY ..................... 2

PREVIOUS INSTRUMENTAL TECHNIQUES .......... 5

DESCRIPTION OF INSTRUMENT . . . . . . . . . . . . 8

DISTINGUISHING FEATURES . . . . . . . . . . . . 17

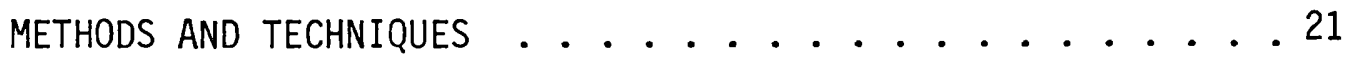

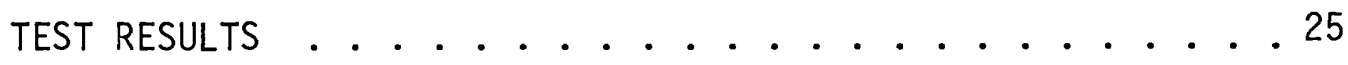

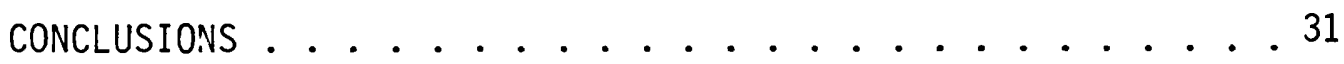

BIBLIOGRAPHY ..................... 33 


\section{LIST OF FIGURES}

1. General System Diagram ............ 4

2. Weber System .............. 6

3. Block Diagram of Instrument .......... . 9

4. Picture of System ............. . . 9

5. Circuit Diagram .............. 11

6. Ellipsoidal Mirror Details ........... 14

7. Block Diagram of Cell ............ 15

8. Picture of Cell ........................ 15

9. Oscilloscope Tracings of System Ambient Light Levels . . . . . . . . . . 23

10. Plot of Fluorescence Concentration . . . . . . . 27

11. Table of Fluorescein Data . . . . . . . . 28

12. Scope Tracing of Polarization Signal . . . . . . 29

13. Plot of Glycerol Curve . . . . . . . . . 30

14. Table of Glycerol Data ............. . 31 


\section{INTRODUCTION AND HISTORY}

Interest in the partial polarization of the fluorescence of macromolecules goes back to Perrin (1) who first published on the subject in 1926. Perrin's theory, based on the assumption that Einstein's equations describing Brownian motion are applicable, focused on the measurement of the rotational relaxation time of molecules as compared to the lifetime of the excited state of the fluorescence. Several workers $(11,12,13)$ in the late 20 's through the mid 30 's showed the lifetime of the excited state of the fluorescence of many dyes in water solution to be of the order of $10^{-8}$ seconds, while the relaxation time of the rotation of these molecules in water is much shorter. Thus almost completely depolarized fluorescence was expected and was observed. Weber (2) in 1952 comments that "On the other hand, fluorescent macromolecules, i.e., proteins, should emit partially polarized fluorescence in water solution as their rotational relaxation times are of much larger orders of magnitude. Measurements of the degree of polarization of the fluorescence should in consequence afford a convenient means of determining the relaxation times of macromolecules in dilute solution under a variety of circumstances." Weber further extended the work to include particles of more than one size carrying the same fluorescent tag.

About this same time papers describing applications of Perrin's and Weber's technique began to appear in the literature, e.g., Laurence (3) "A Study of the Adsorption of Dyes on 'Bovine Serum 
Albumin by the Method of Polarization of Fluorescence", McKay (6) "Effect of Various Environments on the Intrinsic Fluorescence Polarization Spectra of Horse Liver Alcohol Dehydrogenase" (1969) and Watson, et.al. (10) "Polarization Fluoroimmunoassay of Gentamicin" (1976) span the period of the 50's to the present day. Coincident with these application papers additional manuscripts highlighting evolutionary instrument development can be found. Weber (4) published another paper in 1956 on a "Photo-electric Method for the Measurement of the Polarization of the Fluorescence of Solutions". This was followed by others, e.g., Johnson and Richards (5), Rosen (7), Lavorel and Vernotte (8), and Kelly and Dandliker (9). These later reports represented considerable improvement on earlier reported instrument techniques. Further refinement of these instrumentation techniques in terms of simplification and improved sensitivity is the subject of this thesis.

\section{$\underline{\text { THEORY }}$}

Perrin (1) developed the theory of the polarization of fluorescence and published it in 1926. The technique is based on the principle that a molecule tagged with a fluorescent dye, when excited by polarized light, will emit fluorescent light having the same plane of polarization as that of the incident light. However, due to Brownian motion, these tagged molecules, when present in solution, are constantly moving and rotating. Since the fluorescence does not occur until the excited electron decays back into its original energy level and the molecule has continued to rotate during this time, the plane of polarization of the incident and emitted light will differ. 
Although typical excitation lifetimes of many fluorescent dyes in water solution are of the order of $10^{-8}$ seconds, the rotational relaxation times for small molecules are much shorter than this (2). Thus the emitted light from small molecules becomes nearly randomly polarized and may be likened to "electronic noise" or depolarized fluorescence. This is sometimes referred to as "partial" polarization. As the molecules become larger and/or the liquid medium becomes more viscous the rotational relaxation time becomes longer. When it becomes of the order of the fluorescence lifetime, the angular deviation of the plane of polarization becomes small and the measured fluorescence begins to show a preferential polarization - that of the exciting light. Since Brownian motion is directly related to temperature, this too must be considered as a parameter affecting the observed degree of polarization.

An explanation of the technique of measuring polarization is necessary to understand the underlying principle of the apparatus developed. Partial polarization $(p)$ is defined as:

$$
p=\frac{I_{\mid 1}-I_{\perp}}{I_{\|}+I_{\perp}}
$$

Parallel and perpendicular refer to the alignment between the excitation polarizer and the fluorescence polarizers. $I_{1 /}$ and $I_{\perp}$ are the light intensities of the fluorescence measured through polarization filters oriented both parallel and perpendicular to the excitation filter. The system is shown in Figure 1. The excitation beam $I_{1}$ is randomly polarized as indicated by the numerous electric vectors (E) existing throughout al1 360 degrees normal to the axis of the beam. After passing through the interference filter (to establish the preferred excitation wavelength) and the polarizer, the beam is vertically polar- 


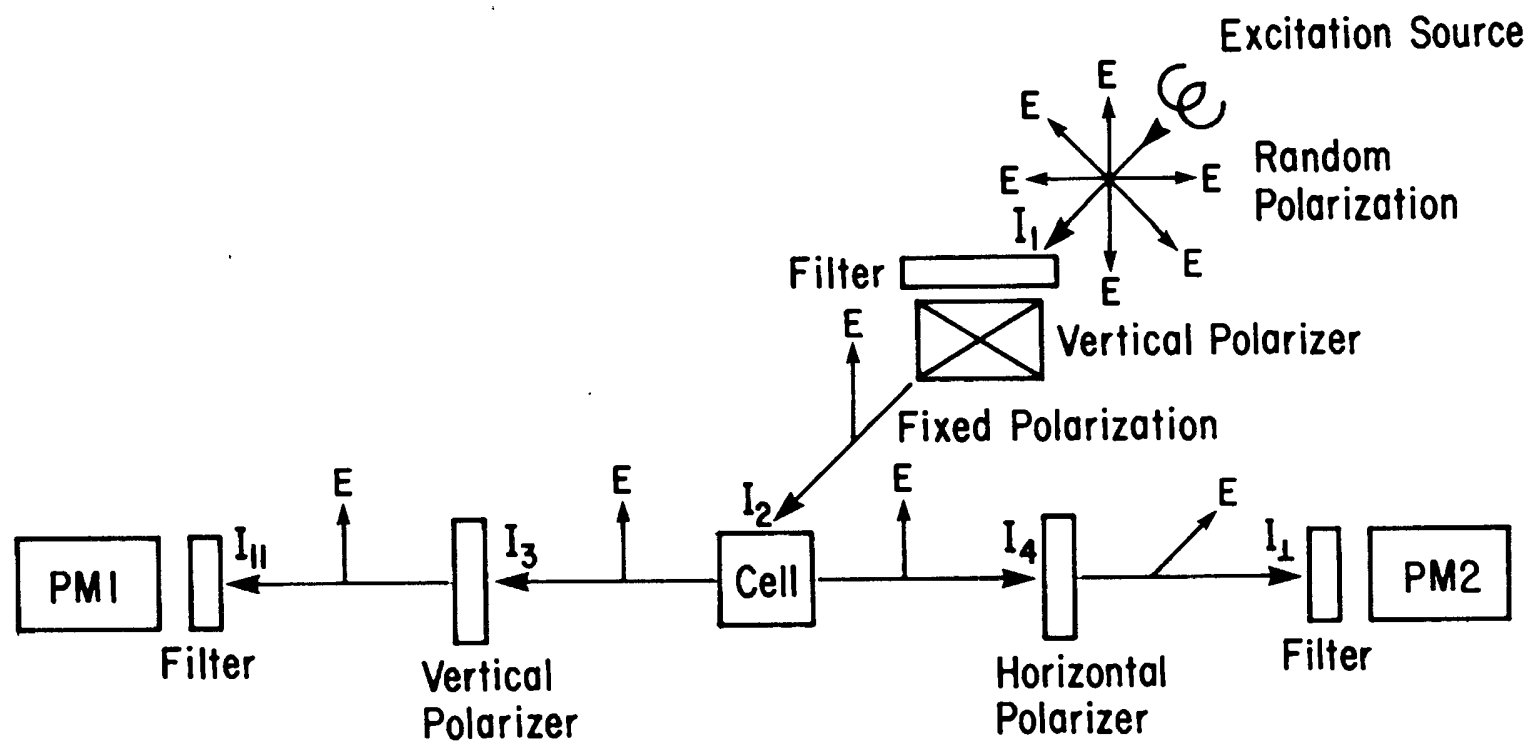

Figure 1. Block diagram showing generalized system for measurement of polarization of fluorescence. Excitation source (upper right) starts out depolarized; is then filtered for appropriate excitation wavelength and vertically polarized before illuminating sample cell. At right angles to the excitation path are two signal photomultipliers; one looking at the vertically polarized fluorescence signal and the other at the horizontally polarized fluorescence signal. Both fluorescence signals pass through light filters establishing the appropriate fluorescence wavelength.

ized as shown by the electric vector being normal to the beam and normal to the axis of the fluorescence beams. The two photomultipliers reading the vertical and horizontal components of fluorescence polarization are positioned on an axis perpendicular to the excitation beam. Not only does this minimize any excitation signal getting to the photomultipliers but it establishes the correct relationship between the fluorescence and excitation polarizers. As shown in Figure 1 the fluorescence signals emanating from the left and right of the cell $\left(\mathrm{I}_{3}\right.$ and $\mathrm{I}_{4}$ ) will be vertically polarized, as indicated by their electric vectors, except for a deviation caused by Brownian motion. $\mathrm{I}_{3}$ passes through a vertical polarizer and a fluorescence filter (selected for 
optimum transmission of the fluorescence wavelength) before impinging on PMI as $I_{11}$.

$\mathrm{I}_{4}$ passes through a horizontal polarizer and an identical fluorescence filter before impinging on PM2 as $I_{\perp}$. If there were no Brownian motion, $I_{11}$ would be maximum and $I_{\perp}$ would be zero, yielding a $p=1$. It should also be noted that if the excitation source were horizontally polarized the fluorescence polarization would be along the axis of the photodetectors, and no light would be seen except for that arising from the depolarizing effects of Brownian motion.

\section{PREVIOUS INSTRUMENTAL TECHNIQUES}

The system that Weber describes in his 1956 paper (4) measures the polarization directly (Figure 2). It consists of a high pressure mercury arc lamp for the excitation source collimated through a series of lenses. The collimated light passes through an excitation filter to set the excitation wavelength and then through a polarizer, which can be rotated between horizontal and vertical polarization, before finally impinging on the sample cell. At right angles to the excitation source are two photomultiplier receivers on a line with each other through the sample cell. On one side of the cell is a reference photomultiplier, fluorescence filter, fixed horizontal polarizer and variable slit. On the other side of the cell, 180 degrees opposed, is the signal photomultiplier, fluorescence filter, fixed vertical polarizer, a variable polarizer and the sample cell. The outputs of the two photomultipliers go to a null-detector galvanometer for current comparison. The measurement technique is as follows:

1. The excitation polarizer is set for horizontal polarization. The variable polarizer is aligned with the fixed polarizer on 
the signal side. The slit on the reference side is adjusted until the signals from the two photomultipliers are nulled.

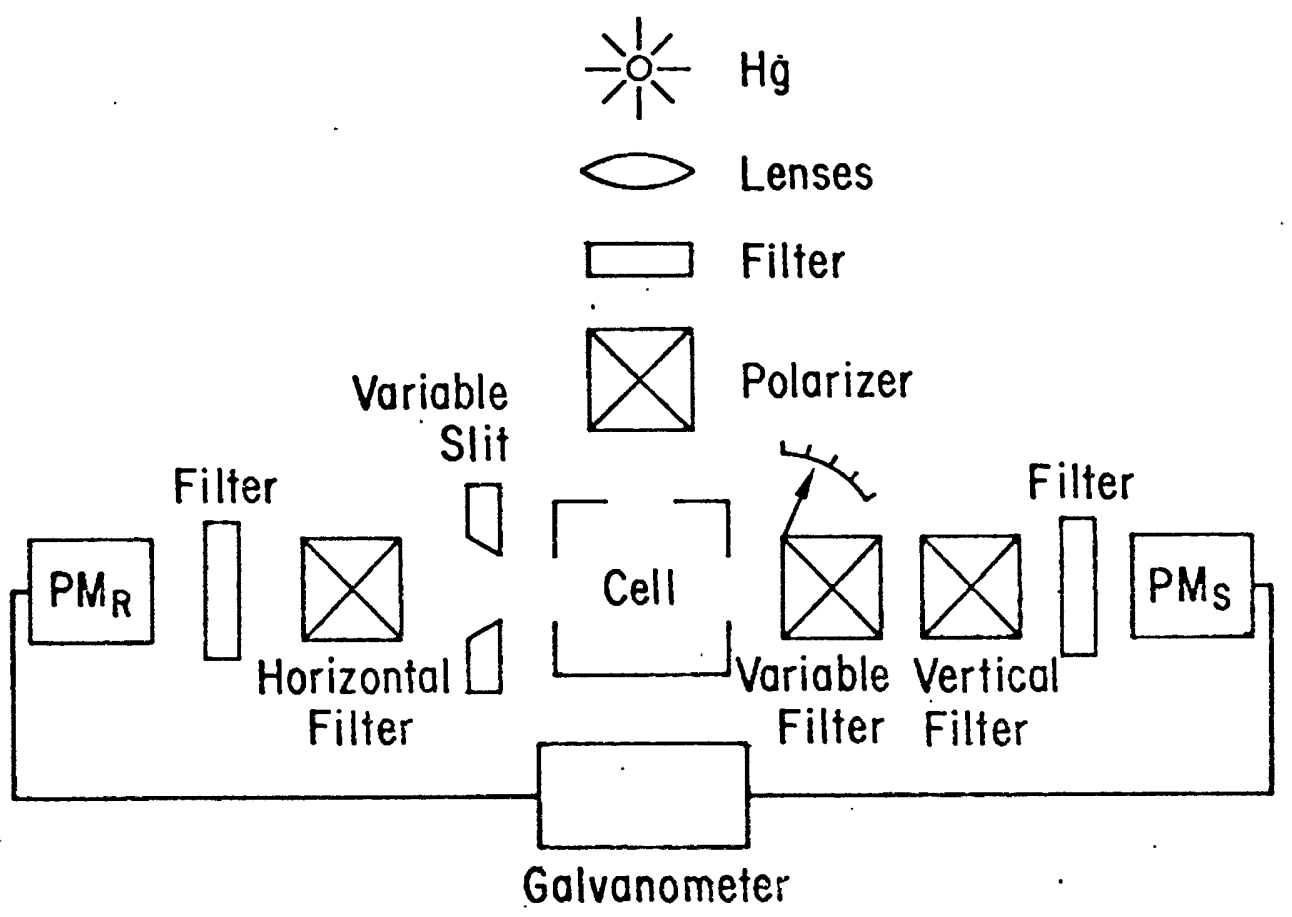

Figure 2. Block diagram of Weber polarization of fluorescence measuring system which reads the polarization angle directly. The excitation path consists of the mercury source, collimating lens, filter and adjustable (vertical and horizontal) polarizer. The reference signal path to the left consists of a variable slit, horizontal polarizer, filter and photodetector. The polarization signal path to the right consists of a continuously variable polarizer, vertical polarizer, filter and photodetector. The outputs of the two photodetectors are compared by a null galvanometer.

2. The excitation polarizer is rotated ninety degrees. This has the effect of switching the signal beam from horizontal polarization to vertical polarization. The reference beam, however, consists of either the horizontal polarization signal, which is a minimum, or the vertical polarization signal attenuated by the horizontal polarizer, also a minimum and 
presumably differing from zero only because of the Brownian motion of the fluorescing molecules, but in either case approximately of the same magnitude.

3. The variable polarizer is now adjusted to restore the nul1. Since the angle of polarization is trigonometrically related to intensity, one can rebalance the two intensities and directly read the angle of polarization from the variable polarizer which is calibrated in minutes of arc. The polarization value is then computed from:

$$
p=\frac{\sin ^{2} \theta}{1+\cos ^{2} \theta \cos 2 \theta}
$$

In 1961 Johnson and Richards (5) reported on a simple instrument using a single photomultiplier and considerably simplified optics. The light source was a mercury lamp with a condensing quartz lens. Between the lens and the sample cell was an excitation filter, polarizer and slit. At right angles to the sample cell was a fluorescence signal light path consisting of the cell with mirrored back walls for increased sensitivity, a fluorescence filter, iris diaphragm, adjustable (zero to ninety degree positions) polarizer, lens, limiting stop, quartz depolarizer and photomultiplier tube. The technique here was to make separate intensity measurements at the zero and ninety degree positions of the polarizer in the signal path. Degree of polarization was then formula determined with the quartz depolarizer eliminating any preferential polarization sensitivity of the photomultiplier tube.

Two-photomultiplier tube techniques were reported on by Rosen (7) in 1970 and advertised as a commercial instrument by SLM Instruments of Urbana, I17inois. Here the two ninety degree to excitation co-axial light paths are used to simultaneously determine vertical and horizontal polarization intensities. The excitation path as in 
previous instruments uses a mercury or xenon light source followed by an excitation filter and fixed polarizer. The two signal paths on a straight line opposed to each other have a fluorescence filter followed by a polarization filter followed in turn by a photomultiplier. In one path the polarizer was parallel to the excitation polarizer and in the other perpendicular to it. Each photomultiplier signal was recorded and degree of polarization calculated by the formula mentioned above. In the SLM Instrument, electronic ratio processing after 10 or 100 (selectable by the operator) samples having been taken provides the polarization factor as a digital display.

McKay (6) in 1969 and Kelly and Dandliker (9) in 1976 added another innovation - that of the rotating polarizer. Here the excitation source was polarized by a fixed polarizer and the fluorescence signal was processed through a rotating polarizer to a single photomultiplier tube. The maximum and minimum signals were taken as $I_{\|}$and $I_{\perp}$ and the polarization value determined by the previously mentioned equation.

Lavorel (8) in 1972 reported a system using a rotating polarizer. However, his rotating polarizer like the system described in this thesis was placed in the excitation path.

\section{DESCRIPTION OF INSTRUMENT}

Figure 3 depicts the overall organization of the instrument described in this thesis. Figure 4 is a picture of the actual system. All components of the system are mounted on $5^{\prime \prime}$ square aluminum plates keyed on a brass tongue attached to a steel base plate for alignment purposes. Critical portions are further held in alignment by two $\frac{1}{4} "$ diameter drill rods passing through two corners of the plates. The 


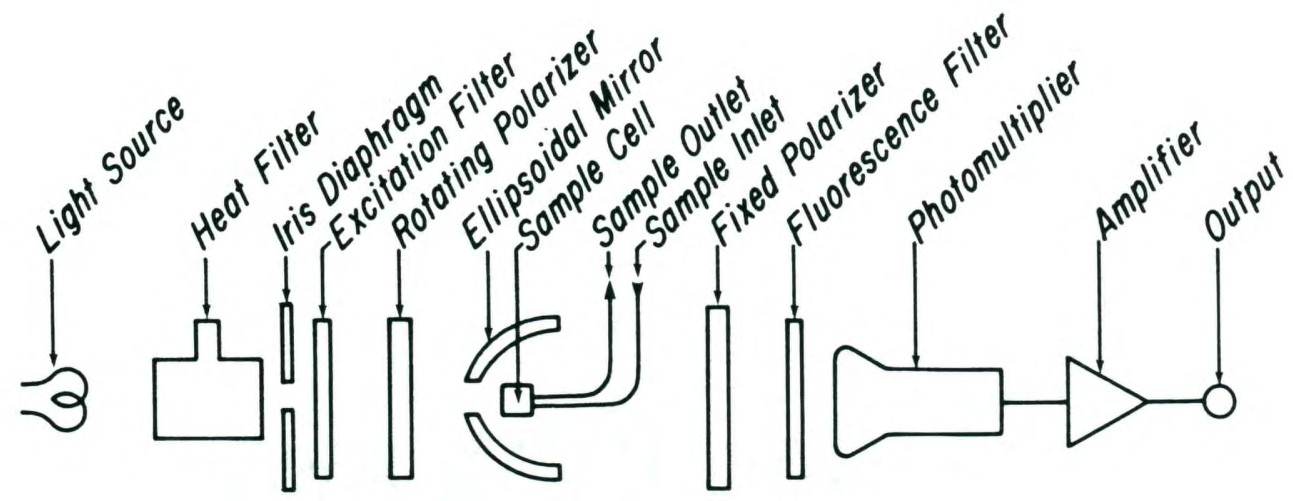

Figure 3. Block diagram of instmonent. Complete optical path

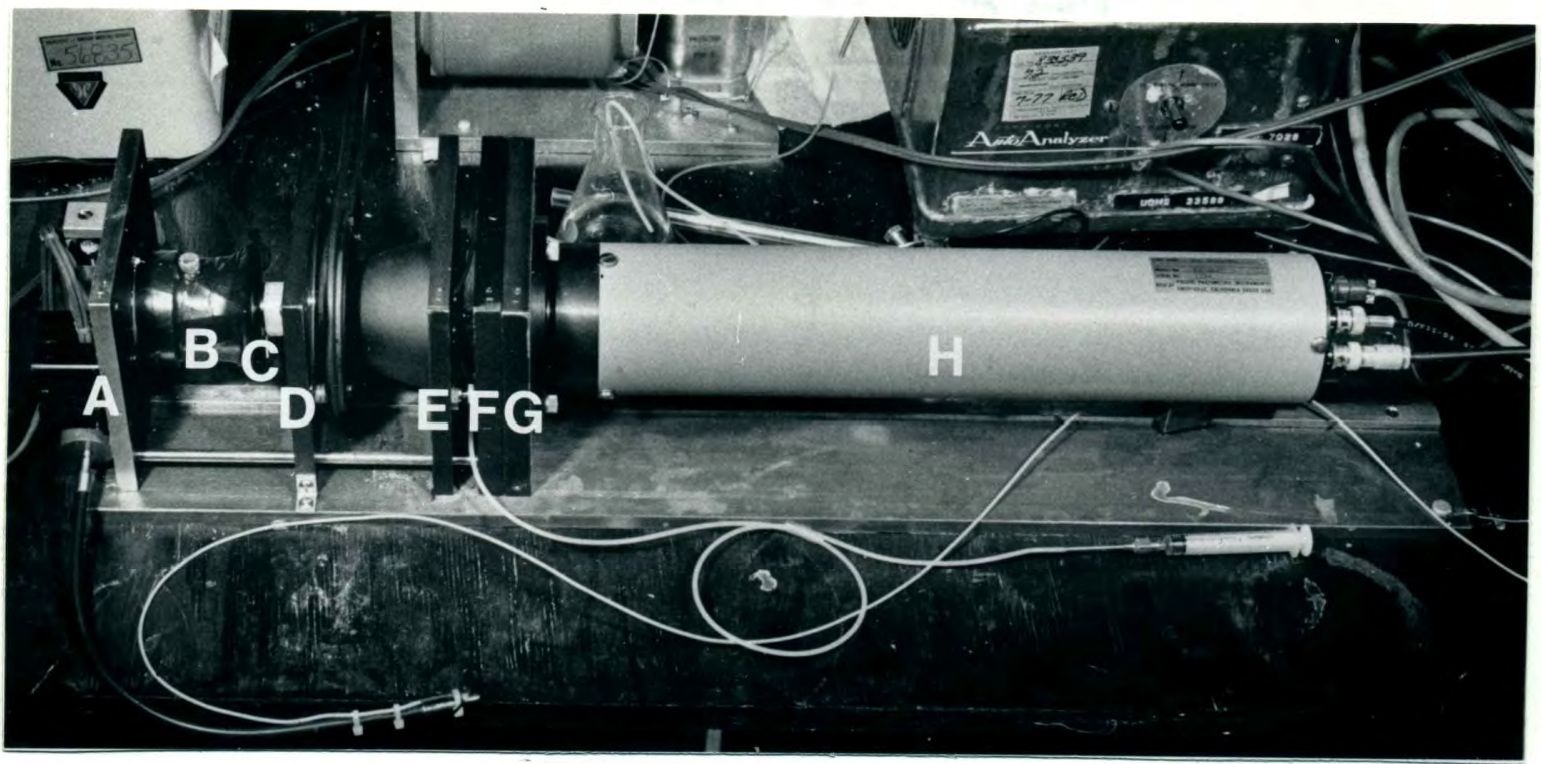

Figure 4. Picture of instmument system. Excitation source (A) is at left and photodetector (H) is at right. Just below the letter (A) is the fiber optic bundle used to isolate the silicon solar cell from the lamp's heat. On the end proximal to the lamp is the 490 nanometer interference filter. 
entire assembly is mounted in a four-foot by two-foot by two-foot lightproof plywood box with an access door on one of the large sides. The entire inside of the box is painted flat black to minimize reflections from the light source as is the metal structure of the instrument itself. Painting of the metal structure was necessary when it was noted that reflections were contributing to polarization of the light source.

At the left end of the system shown in Figure 4 is the light source (A) - a 45 watt quartz-iodide lamp (GE Q6.6AT2 $\frac{1}{2} / \mathrm{CL}$ ) rated at 6.6 amperes. The lamp's intensity is controlled by an electronic servo loop consisting of a solar cell "looking" at the lamp intensity and an operational amplifier comparing that intensity to a regulated voltage. This "looking" provides regulation not only against line and power supply variations but also against lamp aging characteristics. The solar cell is a Hoffman Electronics custom unit consisting of a standard boron-doped silicon solar cell of 1/8" diameter and $1 / 16$ " active area. To prevent the cell from heating, by being too close to the lamp source, an eight inch piece of $1 / 8$ " fiber optics was interposed between the lamp and the solar cell. Between the fiber optic bundle and the $1 \mathrm{amp}$ another 490 nanometer interference filter is interposed to assure that the solar cell sees the same wavelength as the sample cell. Thus regulation is centered on 490 nanometers rather than the much more abundant infrared.

Figure 5 shows the regulator circuit. A CA3140 FET input operational amplifier is used in a follower-with-gain configuration to boost the solar cell signal from approximately $50 \mathrm{mv}$. to 5 volts. The high output impedance of the solar cell is effectively buffered by this amplifier. The 10K potentiometer between pins 1 and 5 of the 


\section{LIGHT CONTROL CIRCUIT}

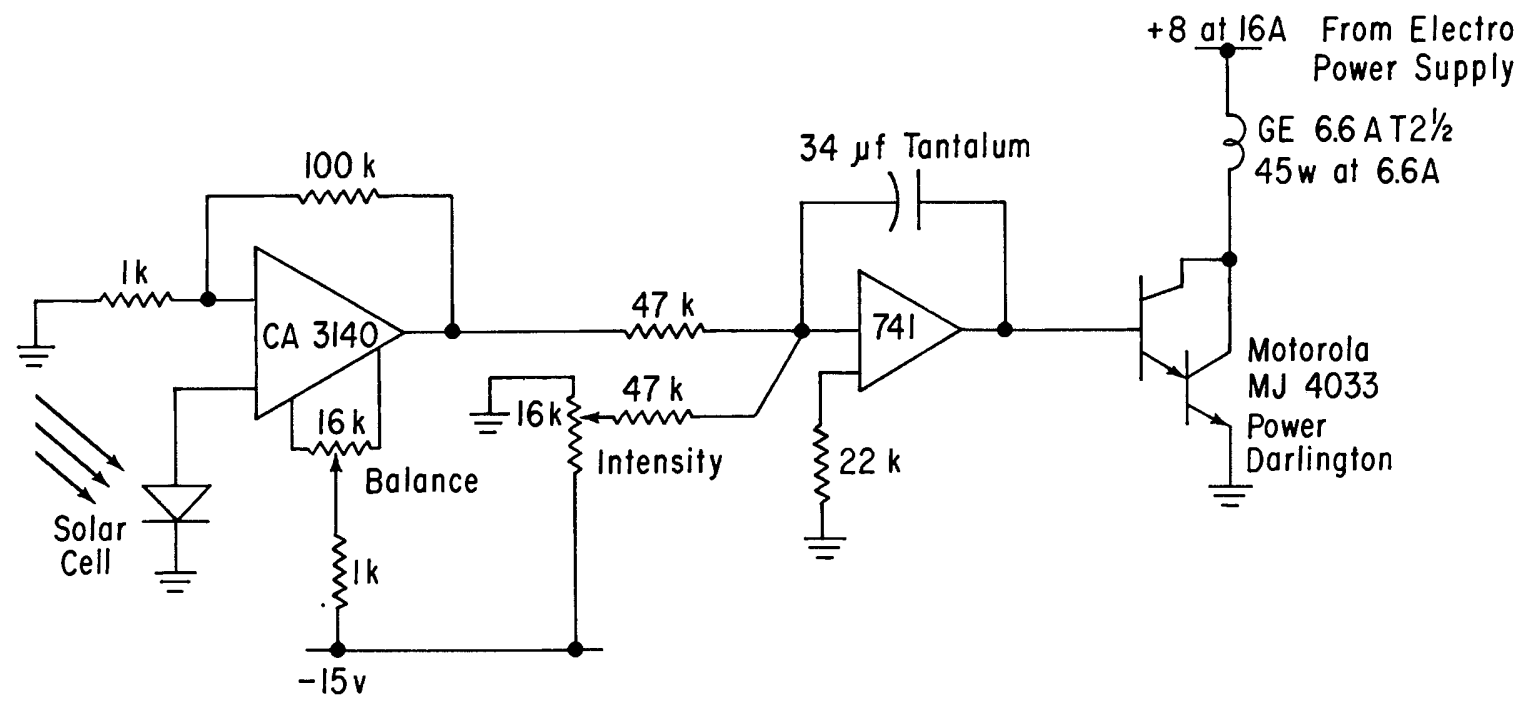

Figure 5. Circuit diagram of Zamp intensity control.

CA3140 allows dark current zeroing of the solar cell. Lamp intensity is controlled by the 741 operational amplifier which is wired as an integrating comparator. The amplified solar cell signal seen through the $47 \mathrm{~K}$ resistor is compared with the reference signal from the $10 \mathrm{~K}$ "Intensity" potentiometer seen through another 47K resistor. Any difference is integrated on the $34 \mathrm{mfd}$. tantalum capacitor to prevent changing the light control voltage more rapidly than the lamp can respond - thus preventing oscillation of the lamp control signal. The 741's output is amplified by the Motorola MJ4033 Darlington power transistor to the appropriate driving current for the lamp ( 6.6 to 7 amperes). The power supply for the Darlington lamp circuit is a separate heavy-duty Electro Power Supply designed for battery charging but possessing filter capacitors for smoothing.

The CA3140 and 741 circuitry are breadboarded on an E\&L Instruments Adam System breadboard which is complete with required \pm 15 volt 
power supply. The MJ4033 power Darlington is mounted on a heat sink attached to the rear of the Electro Power Supply cabinet.

Directly in front of the lamp and attached to the opposite side of the lamp plate is a cell with quartz end plates [Figure 4, (B)] filled with a one-molar copper sulphate solution acting as a heat filter. The cell path length is $50 \mathrm{~mm}$ and the diameter $46 \mathrm{~mm}$ giving a solution volume of $83 \mathrm{ml}$. Originally a smaller cell was tried with a solution volume of only $6.3 \mathrm{ml}$. The heat of the lamp quickly brought this small volume to a boil. Attached to the right front of the heat filter (as seen in Figure 4) is an adjustable iris diaphragm (C) which allows for stopping down the light to only that needed to fill the mirror aperture, thus minimizing stray light in the system. Tests showed that there was an excess of stray light from the source getting into the photomultiplier and to reduce this light a hood was installed over the light source and the heat filter cell was wrapped with black tape.

The next 5" plate to the right (D) houses the excitation filter and the rotating polarizer. The excitation filter is a corian multilayer oxide interference filter (SS4900) transmitting at 490 nanometers. Peak transmission is $50 \%$ with a 10 nanometer half bandwidth. Upper and lower blocking regions have a transmission of $1 \times 10^{-4}$. The polarizer is a 2" $\times 2$ " piece of plastic film available from the Polaroid Division as type HNP'B Linear Polarizer for ultraviolet radiation. At 490 nanometers it has a transmittance of approximately 0.6 and at 550 nanometers a transmittance of approximately 0.4 . In the blocking orientation these transmittances are approximately $3 \times 10^{-5}$ and $2 \times 20^{-5}$ respectively. Rotation is accomplished by a Superior Electric Slo- 
Syn motor driving the rotating member of the 5 " plate via an 0-ring belt. The $72 \mathrm{rpm}$ speed of the motor is stepped down to approximately $0.5 \mathrm{rpm}$ for the rotating polarizer.

The third plate to the right has the ellipsoidal mirror (E) attached to it. This is a Melles Griot product number 02 REM 001 mirror. It has the property of reflecting light originating at the primary focus within the mirror and refocusing it at the secondary focus without altering the polarization. There was some concern about the metallized ellipsoidal surface of the mirror altering the polarization angle. According to Strong (14) the reflection of polarized light from plane dielectric reflecting surfaces is only altered by a 180 degree phase shift. In the case of metallized reflecting surfaces such as the ellipsoidal mirror, which is Rhodium coated, Jenkins and White (15) point out that such surfaces produce an ellipsoidal polarization consisting of $\mathrm{p}$ and $\mathrm{s}$ components. Because of the symmetry of the ellipsoidal mirror, however, the s components cancel out leaving only the $\mathrm{p}$ component with a 180 degree phase shift much as in dielectric reflectors. The mirror has an overall length of $58 \mathrm{~mm}$ and a $12.7 \mathrm{~mm}$ aperture at its rear to admit the excitation light. The front face of the sample cell is mounted at the primary focus which is $12.7 \mathrm{~mm}$ back from the aperture. Details of the mirror are given in Figure 6 , a reproduction of the data sheet.

Details of the sample cell are given in Figure 7 and Figure 8. It consists of a 304 stainless steel cylinder with 0-rings around its body to hold a glass cup in place. The end of the glass cup is a quartz window cemented to the cup body which is made from glass tubing. With the cup in place there is a $1 \mathrm{~mm}$ gap between the front of the stainless steel body and the rear of the cup window forming a 

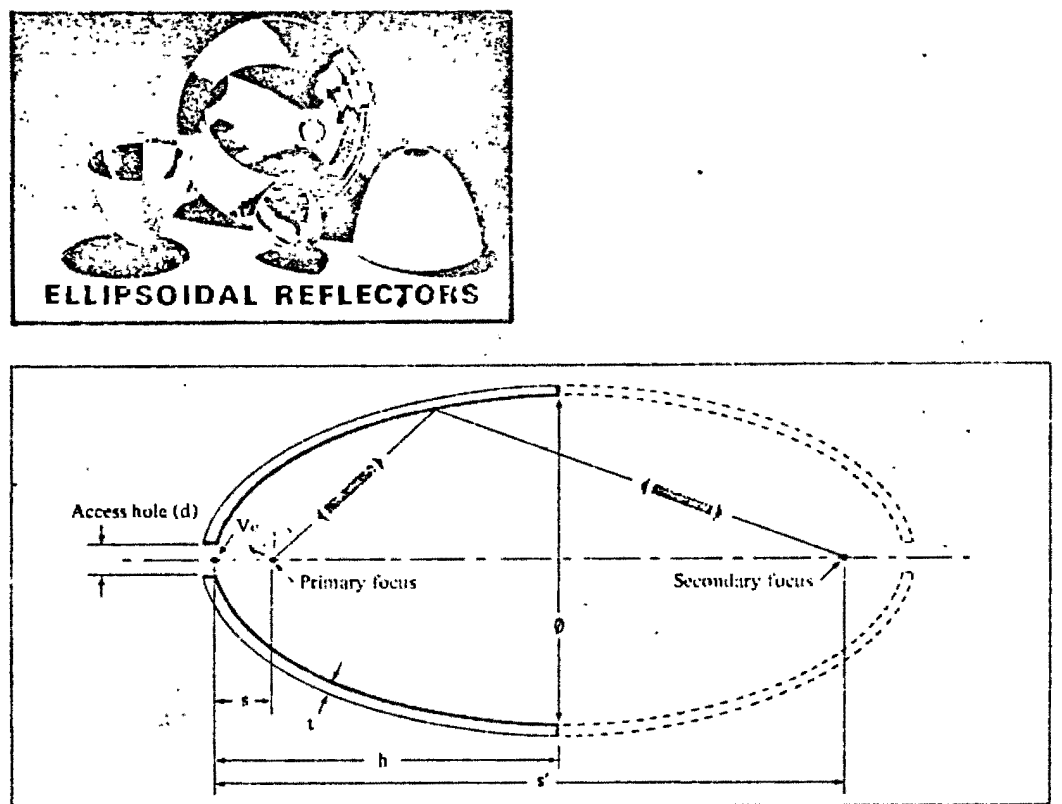

ELLAPSOTDAL REFLECTOK. Kays passing thruugh either focus must after reflection, alse pass through the other. The foci are conjugate points, and the imaging is stigmatic. Stigmatic conjupate distances $s$ and : are

\section{SPECIFICATIONS: ELLIPSOIDAL REFLECTORS}

$$
\begin{aligned}
& \text { s, } t, \text { and } d: \pm 0.13 \mathrm{~mm} \quad 0: \pm .25 \mathrm{~mm} \\
& \text { h. }(0+2 \mathrm{t}): \pm 0.40 \mathrm{~mm} \quad \text { s: } \pm 0.80 \mathrm{~mm} \\
& \text { Maximum separation of optical and meshanical axes at } \\
& \text { vertex: } \pm 0.13 \mathrm{~mm} \\
& \text { Maximum angular devistion of reflected ray from ideal } \\
& \text { parth: } 2 \text { minutes of are } \\
& \text { Maximum Operating Temperature: } 260^{\circ} \mathrm{C}\left(500^{\circ} \mathrm{F}\right)
\end{aligned}
$$

cabulated (wishuut refard to any sign convention) below. These are often sultcd fucal kengths. The pusitions of the foci are best deterin ined experimentally.

Reflectance: Sec table for rhodium earlier in this section for nominal values. klectroformed coating reflecunces

\begin{tabular}{|c|c|c|c|c|c|c|c|c|}
\hline$\stackrel{s}{(m n i)}$ & $\begin{array}{c}* \\
(\mathrm{~mm})\end{array}$ & $\stackrel{h}{(n ! n 1)}$ & $\begin{array}{c}s^{\prime} \\
(\mathrm{mrn})\end{array}$ & $\begin{array}{l}s^{\prime}-s \\
\text { (mni) }\end{array}$ & $\underset{(\mathrm{mm})}{\mathrm{d}}$ & $\begin{array}{c}\text { t } \\
(\mathrm{mm})\end{array}$ & $\begin{array}{c}\Omega \\
\text { (sphicres) }\end{array}$ & $\begin{array}{l}\text { PKODUC: } \\
\text { NIMABEK }\end{array}$ \\
\hline 12.7 & 75 & 58.0 & 102 & $6 \times .9$ & $d 2.7$ & .51 & 83 & O2 REप fNM \\
\hline $\begin{array}{r}12.7 \\
-20.6\end{array}$ & $\begin{array}{r}75 \\
143\end{array}$ & $\begin{array}{r}57.2 \\
105.0\end{array}$ & $\begin{array}{l}102 \\
2+1\end{array}$ & $\begin{array}{r}88.9 \\
221.0\end{array}$ & $\begin{array}{l}25.4 \\
31.8\end{array}$ & $\begin{array}{l}.51 \\
.58\end{array}$ & .68 & $\begin{array}{l}\text { 02 REM OUS } \\
\text { 02 REM } \mathrm{R}, \mathrm{1}\end{array}$ \\
\hline 33.8 & 254 & $18+0$ & 508 & 474.0 & 50.8 & .89 & .76 & O2 REMI 016 \\
\hline 33.8 & 254 & 183.0 & $50 \%$ & 474.0 & 57.2 & 89 & .74 & $02 \mathrm{RH}: \mathrm{N} 1123$ \\
\hline 33.8 & $25+$ & 181,0 & $5 \mathrm{th}$ & $47+.0$ & 63.5 & .89 & .71 & (12 KEA $\mathrm{NZK}$ \\
\hline
\end{tabular}
may be a few pereenr lower than these published values, which were decermined under ideal conditinas for varuum-deposiced films.

Costing durability: Bright rhodium meets or exceeds all applieabic military and DIN specifications.

\section{EL.LIPSOIDAL REFLECTORS}

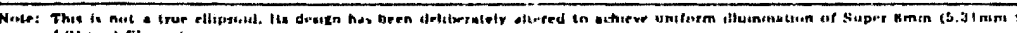
mm) film estr. 


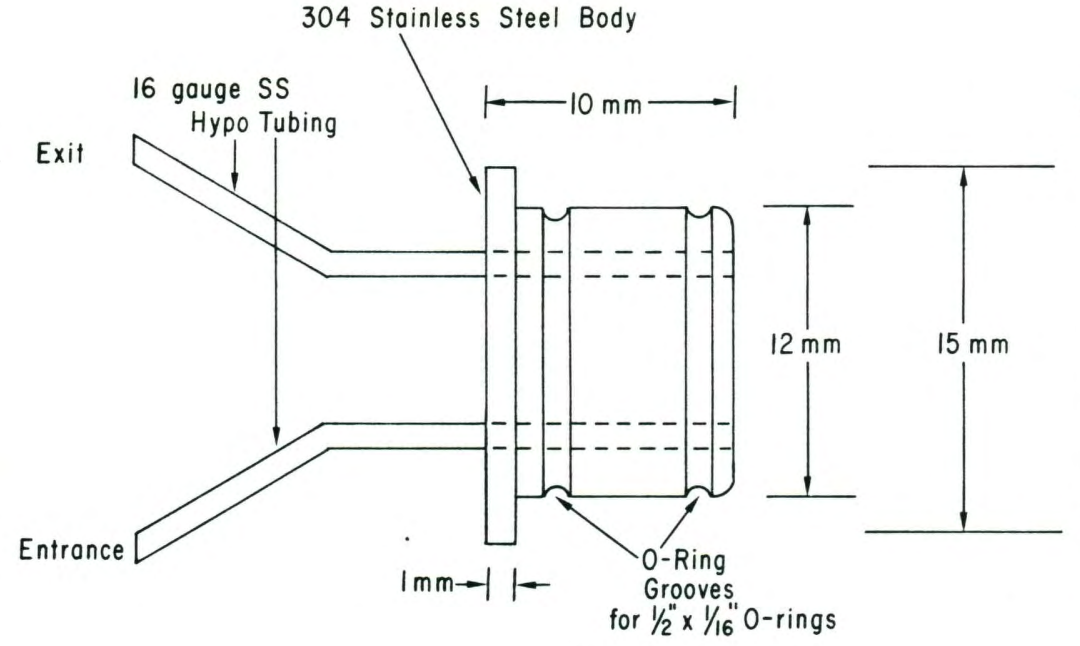

$1-10 \mathrm{~mm} \longrightarrow$

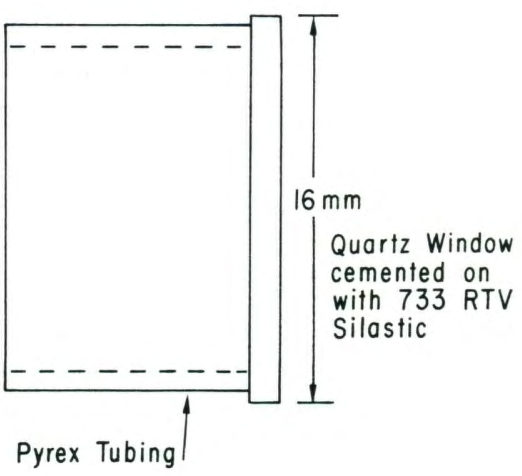

Figure 7. Block diagram of sample cell.

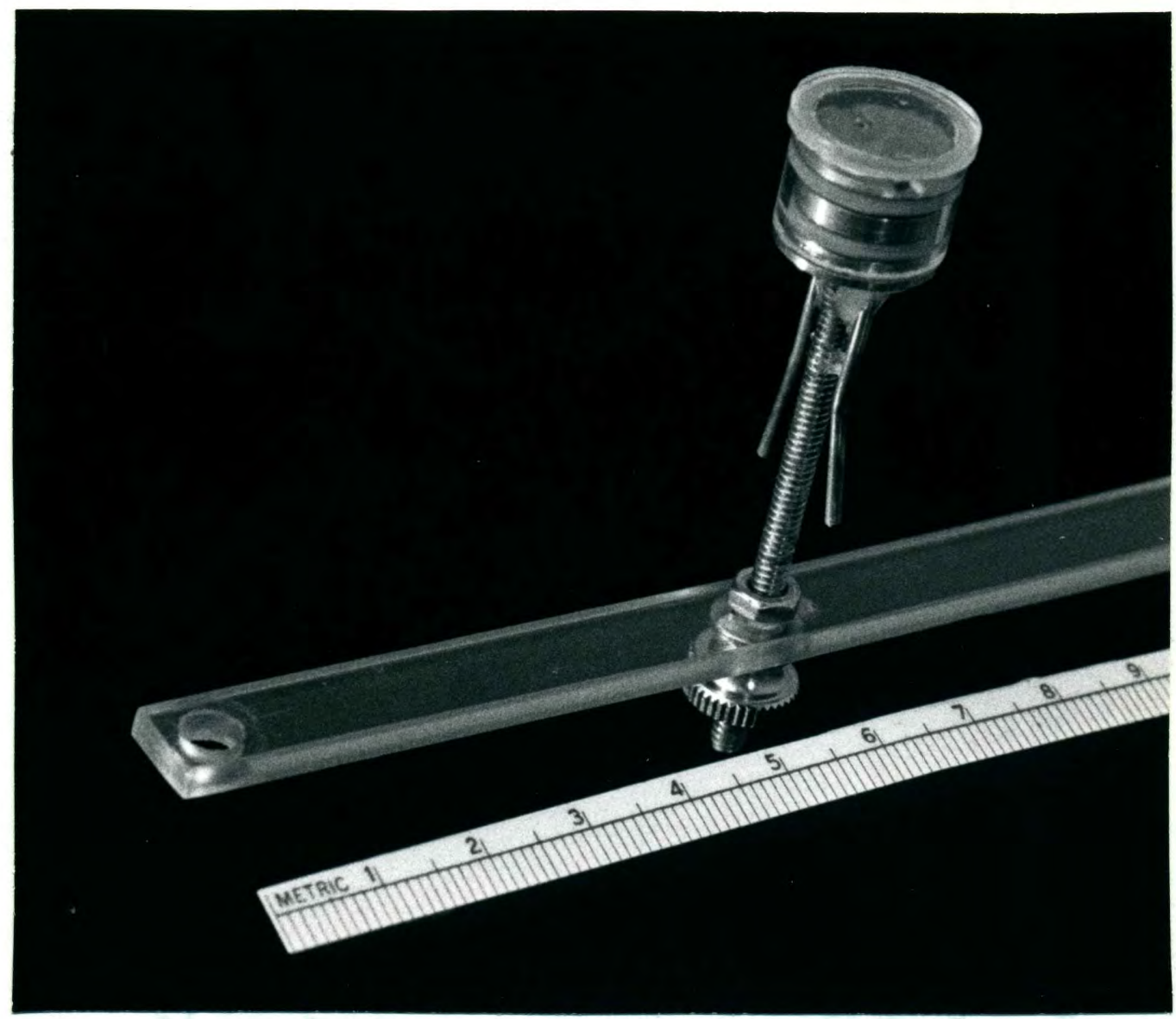

Figure 8. Picture of sample celz. 
$250 \mu 1$. sample space. The sample is injected through a port at the bottom and removed through a port at the top. Connection to these two ports is by 16 gage hypodermic tubing projecting from the rear of the stainless body. A 6-32 brass stud projects from the center of the stainless body and is attached to a plastic bar. This bar is then attached by thumbscrews to the $5^{\prime \prime}$ plate to mount the cell in position. The 6-32 screw allows positioning of the cup face at the primary focus .

The fourth $(F)$ and fifth $(G)$ plates are bolted together as an assembly. The fourth plate contains the fixed polarizer of the same size and material as the rotating polarizer. The fifth plate has the photomultiplier and fluorescence filter $(H)$ attached to it. The fluorescence filter is also a Corian interference filter (SS5500) transmitting at 550 nanometers and having blocking and transmitting characteristics similar to the $\$ 54900$. It is installed in the photomultiplier housing. The photomultiplier detector is a Pacific Photometric Instruments Model $62 / 3 \mathrm{~A} 27$ with eleven current ranges from $10^{-6}$ through $10^{-11}$ amperes in a 10-3-1 sequence and a built-in amplifier. The PM tube itself is an EMI 9635.

The built-in photomultiplier amplifier is an operational amplifier in a current-to-voltage conversion circuit. It also has five ranges of offset capability. to allow cancellation of dark current and ambient light, thus allowing full dynamic signal range. A Pacific Photometric Instruments Model 225 zero to two kilovolt power supply provides high voltage to the photomultiplier tube and an existing dual low voltage laboratory supply provides the \pm 15 volts for the tube's amplifier section. 


\section{DISTINGUISHING FEATURES}

Previously reported systems $(4,5,6,8,9)$ were reviewed before the development of the present system. It was felt that a simpler, more sensitive, more reliable and more easily used system could be developed utilizing modern technology. The several objectives to be achieved were: simplicity, reliability, ease of use and suitability for packaging into a compact, efficient laboratory instrument. Sensitivity is still limited and ease of use can be enhanced even further with the addition of a microprocessor for signal processing. The distinguishing features of the present system are:

1. A 45 watt quartz-iodide tungsten light source.

2. Electronic regulation of the light source by direct monitoring of the light through an interference filter and solar cell combination.

3. Polarization shift by rotation of the excitation polarizer.

4. Enhancement of sensitivity by utilizing an ellipsoidal mirror to reflect the fluorescence signal to the photomultiplier.

5. Use of a fixed fluorescence polarizer in front of the photomultiplier which eliminates the effect of preferential polarization of the photomultiplier.

6. The use of film type UV polarizers utilizing much less space than more expensive crystal polarizers.

7. The use of a small, flow-through sample cell assembly requiring less than $250 \mu 1$. of sample. Samples are injected directly by syringe.

8. The instrument is a small (less than four feet long) 
assembly with a colinear light path. The basic system is $5^{\prime \prime} \times 5^{\prime \prime}$ square. Even with the required power supplies and light regulator it can easily be packaged into an efficient assembly requiring less laboratory space than many conventional instruments.

9. The system is designed to have maximum light bandwidth and at the same time maximum optical simplicity. The light source has reasonable output down to 320 nanometers and of course extends into the infrared region. A copper sulphate heat filter with quartz end windows eliminates much of the infrared while passing light into the UV region. Collimating and focusing optics which would have to be quartz for passing UV signals are eliminated. Only the iris diaphragm is included to eliminate excess stray light and hence "noise" in the system. The polarization filters are specified to transmit down to 275 nanometers. Also the ellipsoidal mirror, which is a Rhodium plated front surface mirror, is specified to have an absolutely flat $80 \%$ reflectance from 375 nanometers to 700 nanometers.

Taking the distinguishing features of the system by number and in order, comments on the system are:

1. The 45 watt quartz-iodide tungsten light source has the advantage of minimizing the problems of size and heat. It is also easier to regulate than mercury-arc and xenon pressure sources. However, the relatively low intensity compared to reported systems with 150 watt and 450 watt mercury and xenon light sources inhibits sensitivty despite 
the gains made by the use of the ellipsoidal mirror. Later development to improve sensitivity may require a higher wattage light source if existing signal to noise ratio cannot be improved.

2. Electronic regulation of a low voltage tungsten lamp is quite easy. However, if it becomes necessary to utilize higher wattage mercury or xenon sources the electronic regulation and the power supply will become more sophisticated and bulky.

4. The ellipsoidal mirror utilized in a colinear optical path improves the sensitivity of the system by capturing more fluorescence signal. However, it will be shown in the test results that surface reflections of the excitation light from the front face of the sample cell do contribute to a poor signal to noise ratio. An anti-reflective coating for the quartz window will be sought to reduce this problem.

3,5. Placing the rotating polarizer in front of the excitation source and the fixed polarizer in front of the photomultiplier eliminates any polarization preference of the photomultiplier. However, it also assumes that polarization of the light source is zero or minimal - not an entirely valid assumption. Some polarization of the light source exists (see TEST RESULTS). However, the magnitude of the polarization is small compared to the noise signal and its resulting polarization signal which exists in the basis system. Some of the ambient noise signal comes from the $4 \%$ surface reflection of the sample cell. 
6. The polarization material is Polaroid's HNP'B linear polarizer film suitable for use down to 275 nanometers. This material is much less expensive and bulky than Glan-Thomson prisms and other crystal polarizers.

7. The sample cell is ideal for volume work such as that of the Clinical Pathology Laboratory at the University of Oregon Health Sciences Center. It requires minimal volume (less than $250 \mu \mathrm{l}$.) and the samples clear readily when fresh injections from a syringe are made (see TEST RESULTS). However, sample temperature control is very important - to within 0.1 or 0.2 degrees $C$. Two methods are under consideration for achieving this: 1) Modification of the back of the sample cell to incorporate a water jacket and pumping temperature stabilizing water into this jacket or 2) Addition of a thermoelectric heat pump to the back of the cell. The latter is preferred for simplicity and efficiency but the cost of acquiring such a unit specifically designed for this configuration might be prohibitive.

8. The original goal was to develop a system which was small and efficient; the arrangement of the colinear light path employing an ellipsoidal mirror served these purposes. Addition of the required motor drive for the rotating polarizer, power supplies for the photomultiplier and electronic drive for the lamp do not compromise the original concept. Readout, whether it be through oscilloscope (as present) or through microprocessor and digital printout (anticipated for further development) do not compromise this basic package as they can be quite small and efficient auxiliary packages. 
9. Elimination of focusing and collimating lenses considerably simplifies the system and allows for maximum light bandwidth. The bandwidth becomes important when excitation wavelengths are required below 400 nanometers. Fluorescence wavelengths are not required in this region, but the focusing characteristics of the ellipsoidal mirror negate the requirements for focusing lenses and simplify the optical system.

\section{METHODS AND TECHNIQUES}

Several types of tests were conducted to verify the operating characteristics of the instrument. The first test was to measure the instrument's sensitivity to fluorescein concentration and its linearity of response to varying concentrations. Solutions of fluorescein in 0.1 molar sodium phosphate buffer were prepared. Concentrations were prepared in decade steps from $10^{-6}$ molar to $10^{-11}$ molar. These samples were run with the rear polarizer removed and the front polarizer stationary but rotated for maximum response (an indication that some polarization existed in the light source). The photodetector signal was displayed on a Tektronix 7633 storage oscilloscope using a 7A22 preamplifier. Pictures of the signal response, stored on the oscilloscope screen, were taken with a Tektronix C-12 camera. The vertical sensitivity and horizontal sweep speed appear at the top of each picture. More than one photomultiplier sensitivity setting was used during each run. To accomodate this the vertical sensitivity of the oscilloscope was multiplied by a factor of one at the highest photomultiplier setting. When the photomultiplier sensitivity was decreased it was decreased by a factor of ten. To accomodate this the vertical oscilloscope sensitivity was multiplied by a factor of ten for proper scaling. 
This gave voltage readings ranging up to 45 volts even though the output swing of the photodetector system was limited to 15 volts. However, the scaling of the actual light intensity was correct. During each oscilloscope trace the photomultiplier high voltage was turned off momentarily half-way through the sweep. This allowed verification of zero during each trace and made later reading easier because the scale markings in the center of the screen were not obliterated by the trace.

The first sample of each series was a 0.1 normal hydrochloric acid wash. This was to wash out any previously left contamination. Each sample consisted of an injection of approximately 6 milliters of fluid to guarantee adequate clearance of the previous sample in the 250 microliter chamber plus the volume of the fill and exhaust tubing. Since acid inhibits fluorescence it also gave a system reading on the basic ambient "noise" level. Each acid injection was followed by an injection of buffer consisting of 0.1 molar sodium phosphate with no fluorescein. This washed out the acid and eliminated the possibility of fluorescein inhibition by the acid. Then the range of samples were injected in increasing order of concentration to minimize possible errors from "tailings" of the previous solution, even though earlier tests with injected food dyes did indicate total clearance with 6 milliters of solution.

Since the basic ambient light level of the system (comparable to electronic "noise" level) was so high it restricted the minimum detectable fluorescent level to a concentration of $10^{-8}$ molar. An attempt was made to determine the major source of the stray light. One source was reflections from the front surface of the sample cell window. These would again be reflected by the ellipsoidal mirror to the photodetector. Black velour paper was used to cover the front surface of the cell and 
the ambient light level measured. The signal under these conditions was compared with that from the uncovered cell filled with an acid solution possessing no fluorescence. Figure 9 shows the results. The top trace is with the cell uncovered and a non-fluorescing acid solution in the chamber. The bottom trace is with black velour paper covering the front window of the cell. As seen from the figure the black paper provided

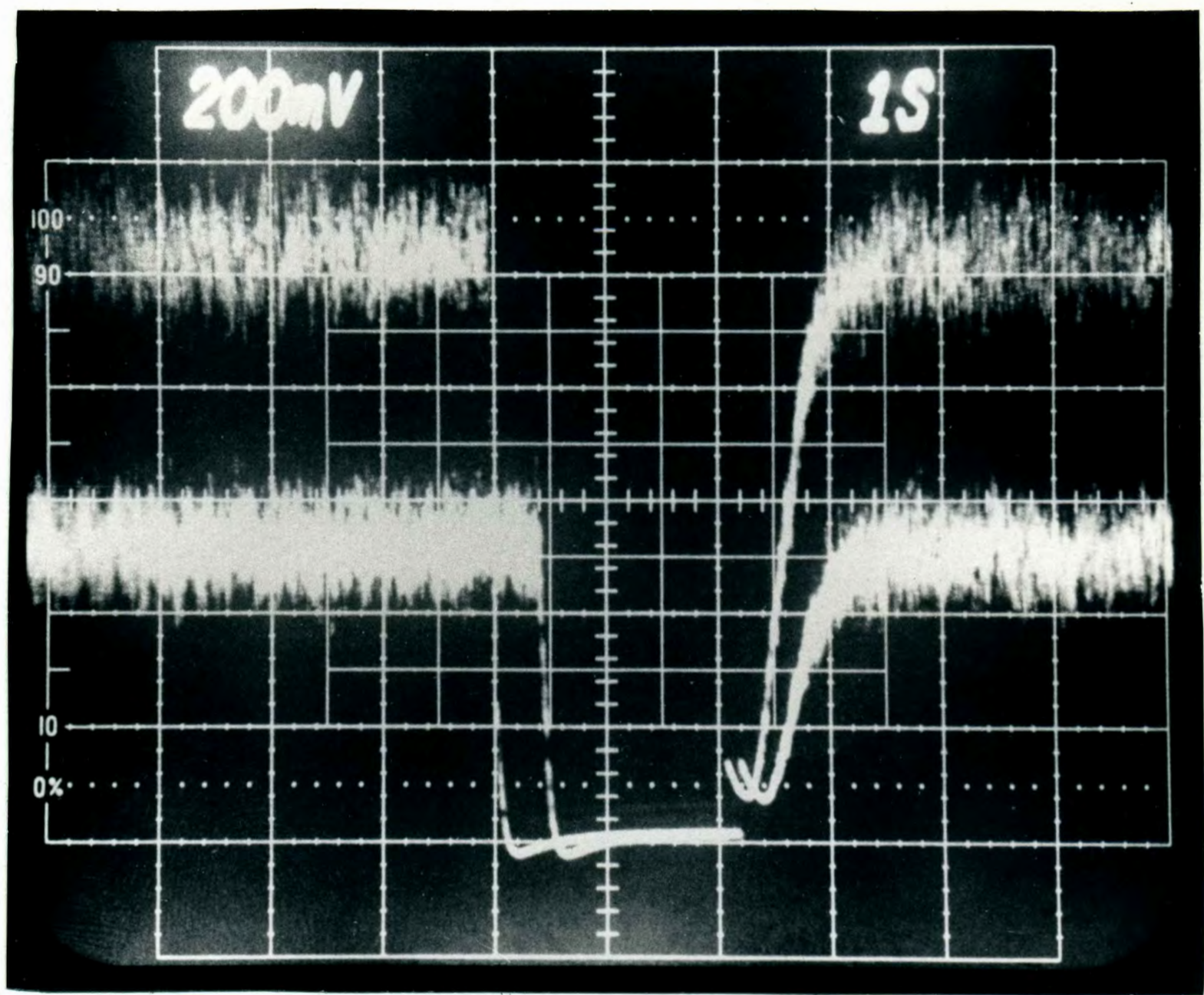

Figure 9. Oscizloscope tracing of system ambient light levels. Upper trace is with cell in normal operation filled with 0.1 molar hydrochloric acid to inhibit fluorescence. Lower trace is with front face of cell covered with black velour paper. Difference between two indicates magnitude of cell front surface reflection of excitation light. Signal zero is $1 \mathrm{~cm}$. from bottom of screen and verified by momentarily turning off photomultiplier high voltage half-way through sweep. 
an almost $50 \%$ reduction in ambient 1 ight. These tests were made with a PM sensitivity setting of $10^{-7} \mathrm{~A}$. The excitation lamp current was $7 \mathrm{~A}$., a value used throughout the experiments.

After the sensitivity and Tinearity of the system to varying fluorescein concentrations were determined, the system was checked for its ability to measure polarization. Solutions of glycerol ranging from $62 \%$ glycerol to $90 \%$ glycerol were prepared with $1 \times 10^{-6}$ molar fluorescein concentration. This high a fluorescein concentration guaranteed a good signal to noise ratio. The rear polarizer was reinstalled and the motor rotating the front polarizer was turned on. The previously described sequence of an acid wash followed by a buffer wash followed by the samples in ascending order of viscosity was followed for each run. Pictures were taken of the oscilloscope signal for each sample point for later analysis. The rotating polarizer produced a sinusoidal signal in time rather than the constant signal obtained in the straight fluorescein sensitivity runs. Values for polarization were calculated from:

$$
\frac{I_{\max }-I_{\min }}{I_{\max }+I_{\min }}
$$

Scaling was done as before with the highest photomultiplier sensitivity used giving a factor of unity to the oscilloscope's vertical amplifier and each $\times 10$ decrease in photomultiplier sensitivity giving a $x 10$ increase to the vertical amplifier sensitivity. Only two photomultiplier ranges were used, $10^{-8} \mathrm{~A}$ and $10^{-7} \mathrm{~A}$.

To reproduce the curve of $1 / p$ vs $T_{K}^{0} / \eta$ as in Lavorel (8) it was necessary to calculate the viscosity, $n$, in poise of the standard solution used. This required the use of a viscometer to determine the time of a calibrated volume to run through a calibrated orifice. Then a pycnometer was used to determine the solution density by weighing a 
calibrated volume. These two readings allowed the calculation of $n$ by:

$$
\frac{\eta_{1}}{n_{2}}=\frac{\rho_{1} t_{1}}{\rho_{2} t_{2}}
$$

The t's are the viscometer times and the $\rho$ 's are the pycnometer densities. Subscript (1) is for the glycerols and subscript (2) is for water.

Temperature instability was a problem throughout the experiments. No provision was made to control the temperature of the sample cell at this stage of development because of the mechanical complexity involved. All work was done at room temperature as rapidly as possible to minimize any temperature change during a run. The temperature was recorded at the beginning and end of each run and typically varied onty two or three degrees centigrade. Nevertheless it is certain that this affected the scatter of the data somewhat as even water has a change in viscosity of the order of $1 \% /{ }^{\circ} \mathrm{C}$. Also the fluorescence in fluorescein is temperature dependent.

\section{TEST RESULTS}

Several tests were performed to assess the performance characteristics and sensitivity of the system. The first was a fluorescein sensitivity test to determine the Tinearity of the response and the ultimate sensitivity of the system. Concentrations of fluorescein in phosphate buffer were made ranging from $10^{-11}$ molar to $10^{-5}$ molar in decade steps. The voltage output of the photomultiplier was read on a Tektronix 7633 storage oscilloscope using a 7 A22 plug-in amplifier. The sweep speed used was $1 \mathrm{sec} . / \mathrm{cm}$. throughout the tests. The first detectable response was at a concentration of $10^{-8}$ molar. It was 
found that system sensitivity is not limited by instrument sensitivity but rather by system "noise" - stray light and reflections in the system. The lamp was maintained at 7 amperes. The photomultiplier was operated with $1.5 \mathrm{kV}$ on the dynodes at a sensitivity setting of $10^{-8} \mathrm{~A}$. Thus three more decades of photomultiplier sensitivity were avai1able and the dynodes could still be raised to $2 \mathrm{kV}$. The other part of this test was for linearity of response to varying concentrations of fluorescein. Concentrations of fluorescein in buffer were prepared ranging from $0.1 \times 10^{-6}$ to $0.9 \times 10^{-6}$. Five runs were made and indeed the system did exhibit excellent linearity. The average of these runs are shown in the plot of Figure 10 and a table of the results including the average and standard deviation for each point is shown in Figure 11. A11 runs were done with the rear polarizer removed and the front polarizer set for maximum response. The fact that there was a variation in response with the angular position of the front polarizer indicated that there was a slight polarization of the light source. This fact was also evident by the value of the (p) numbers when the fluorescein in glycerol concentrations were run in later tests. Each run was preceded and ended by an $0.1 \mathrm{~N} \mathrm{HC1}$ wash to establish a "noise" baseline and system reproducibility as well as cleaning out the system. This was followed by a buffer wash to eliminate the acid residue which would inhibit fluorescence. The system baseline signal ranged between 500 and $600 \mathrm{mV}$. Whereas the fluorescein signal ranged between 4.0 and 45 volts. Rotation of the front polarizer produced no discernable polarization of the acid "noise" signal. Occasionally there were obvious "bad" data points characterized by low response. These were determined to arise from bubbles in 


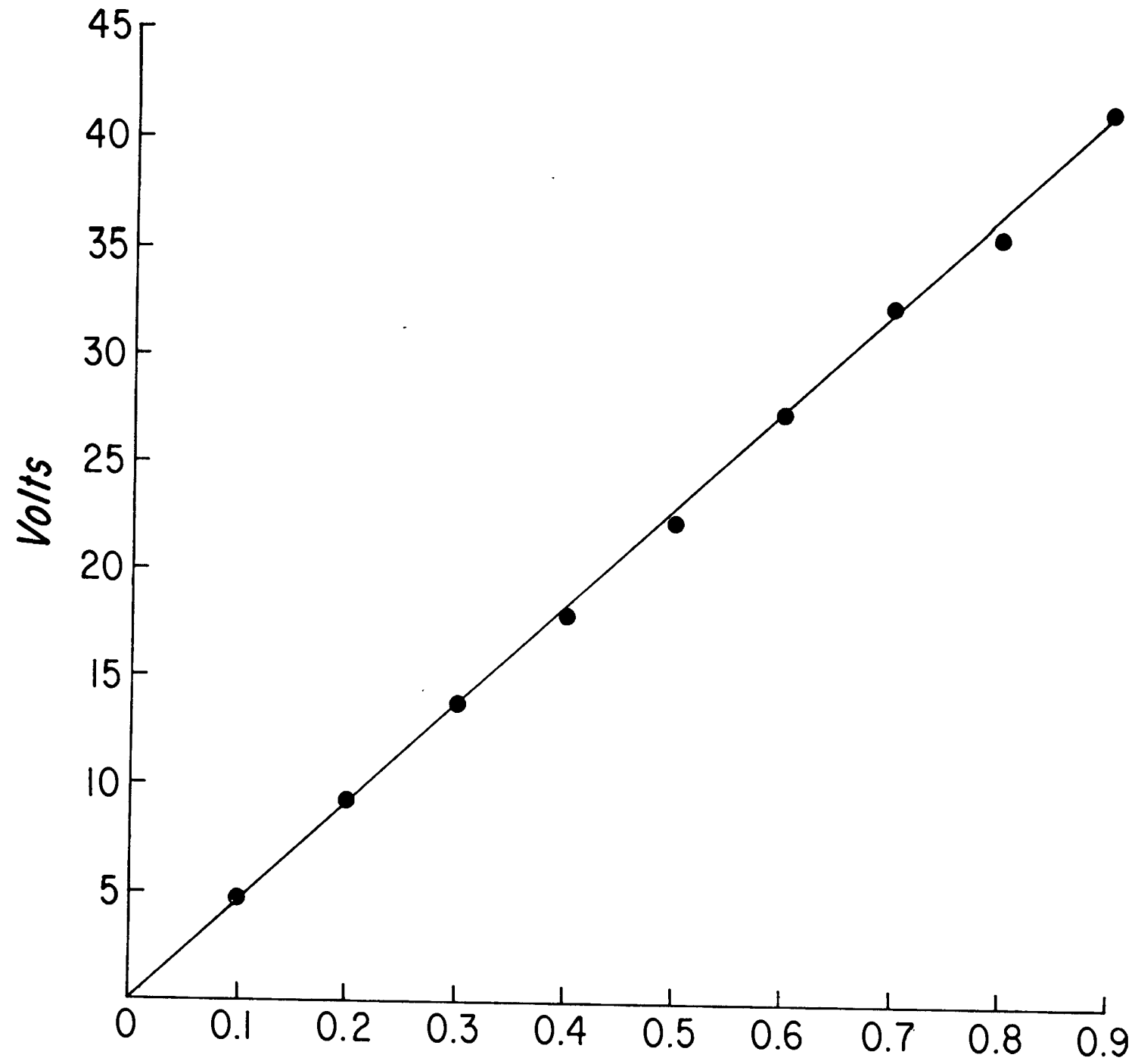

Fluorescein $\times 10^{-6} \mathrm{M}$

Figure 10. Plot of the averages of five mus of fluorescein concentration versus fluorescence signal response in volts.

the sample cell. Reinjection of the sample solution always cleared the bubble and gave an appropriate result.

Tests were made of the linearity of the polarization (p) response and compared with results of a similar experiment by Lavorel (8). A $10^{-6}$ molar fluorescein concentration in various concentrations of glycerol in buffer were prepared. Eight different concentrations ranging from $60 \%$ glycerol to $90 \%$ glycerol were prepared covering the 


\begin{tabular}{|l|c|c|c|c|c|c|c|}
\hline CONCENTRATION & $F_{1}$ & $F_{2}$ & $F_{3}$ & $F_{4}$ & $F_{5}$ & $\bar{x}$ & $s$ \\
\hline $0.1 \times 10^{-6}$ & 4.0 & 4.5 & 5.4 & 4.3 & 5.7 & 4.78 & 0.73 \\
\hline $0.2 \times 10^{-5}$ & 9.6 & 9.6 & 10.0 & 7.6 & 10.4 & 9.44 & 1.08 \\
\hline $0.3 \times 10^{-6}$ & 13.4 & 15.0 & 14.0 & 11.2 & 15.5 & 13.82 & 1.68 \\
\hline $0.4 \times 10^{-6}$ & 15.0 & 19.0 & 18.0 & 16.5 & 21.0 & 17.90 & 2.30 \\
\hline $0.5 \times 10^{-6}$ & 21.5 & 23.5 & 22.5 & 20.5 & 23.5 & 22.30 & 1.30 \\
\hline $0.6 \times 10^{-6}$ & 24.5 & 29.0 & 26.5 & 28.0 & 29.0 & 27.40 & 1.92 \\
\hline $0.7 \times 10^{-6}$ & 30.0 & 33.5 & 31.0 & 34.0 & 34.0 & 32.50 & 1.87 \\
\hline $0.8 \times 10^{-6}$ & 34.0 & 37.0 & 35.0 & 40.0 & 39.0 & 37.00 & 2.55 \\
\hline $0.9 \times 10^{-6}$ & 38.0 & 43.0 & 40.0 & 45.0 & 41.0 & 41.40 & 2.70 \\
\hline
\end{tabular}

Figure 11.' Table of fluorescence signal responses (in volts) versus concentration (molar) for five mus. The average and standard deviations are shown in the last two columns to the might.

range indicated by Lavorel. Again five runs were made and again the system did exhibit excellent linearity of response. Figure 12 shows a typical oscilloscope tracing of the signal with the polarizer rotating. $I_{11}$ is the signal maximum and $I_{\perp}$ is the signal minimum. Zero is one division above the bottom of the screen. $1 / p$ was plotted against $T / n$ and the average of the responses shown in Figure $13 . T$ is in degrees Kelvin and $n$ is poise. Figure 14 is a table of the data collected with the average and standard deviations shown.

A $10^{-6}$ molar fluorescein concentration was picked to agree with the samples used by Lavorel. This also provides a healthy photomultiplier signal with good signal-to-noise ratio. The values of $(p)$ were somewhat smaller than those reported by Lavorel. Thus where Lavorel's graph of $(1 / p)$ runs from 0 to 5 , Figure $13^{\prime}$ 's abscissa runs from 0 to 18. This arises from the presence of horizontal polarization of the light source mentioned before. This polarization yields a higher than normal $I_{\perp}$ making $(p)$ somewhat smaller than it should be. Nevertheless this constant offset of $I_{\perp}$ does not destroy the linearity of the system response. 
Early in the data collection stage attempts were made to determine the effect of temperature on fluorescein sensitivity and polarization values. Within the limits of variability of room temperature (22 to 25 degrees C.) there was no observable effect on readings. Data runs were made quickly enough that system temperature did not vary more than 0.5 degrees $C$.

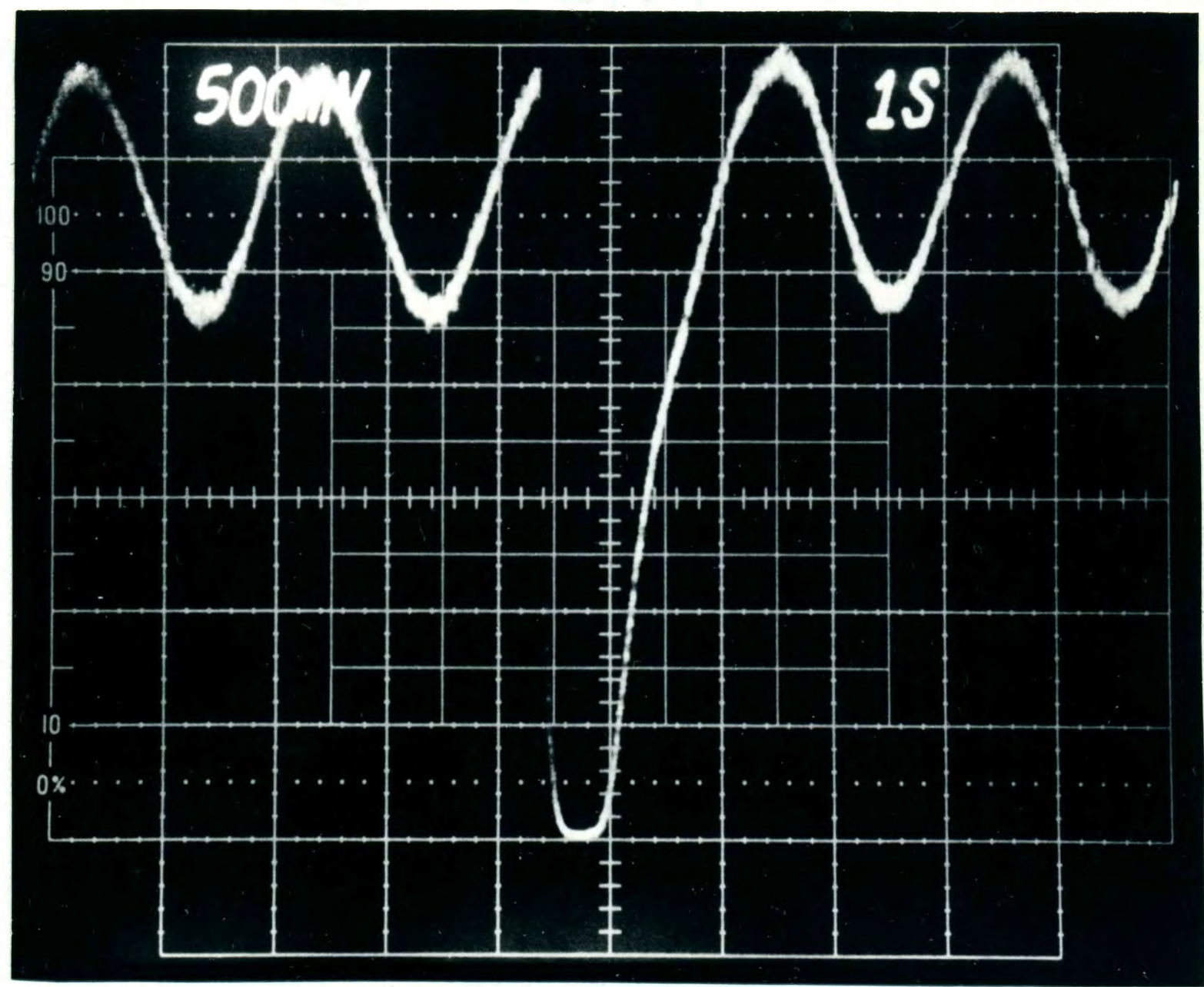

Figure 12. Typical oscilloscope tracing of the polarization signal. I I is taken as the maximum of the sinusoidal signal and $I_{\perp}$ as the minimum. Signal zero is $1 \mathrm{~cm}$. from the bottom of the screen and verified by momentarily turning off photomultiplier high voltage half-way through sweep. 


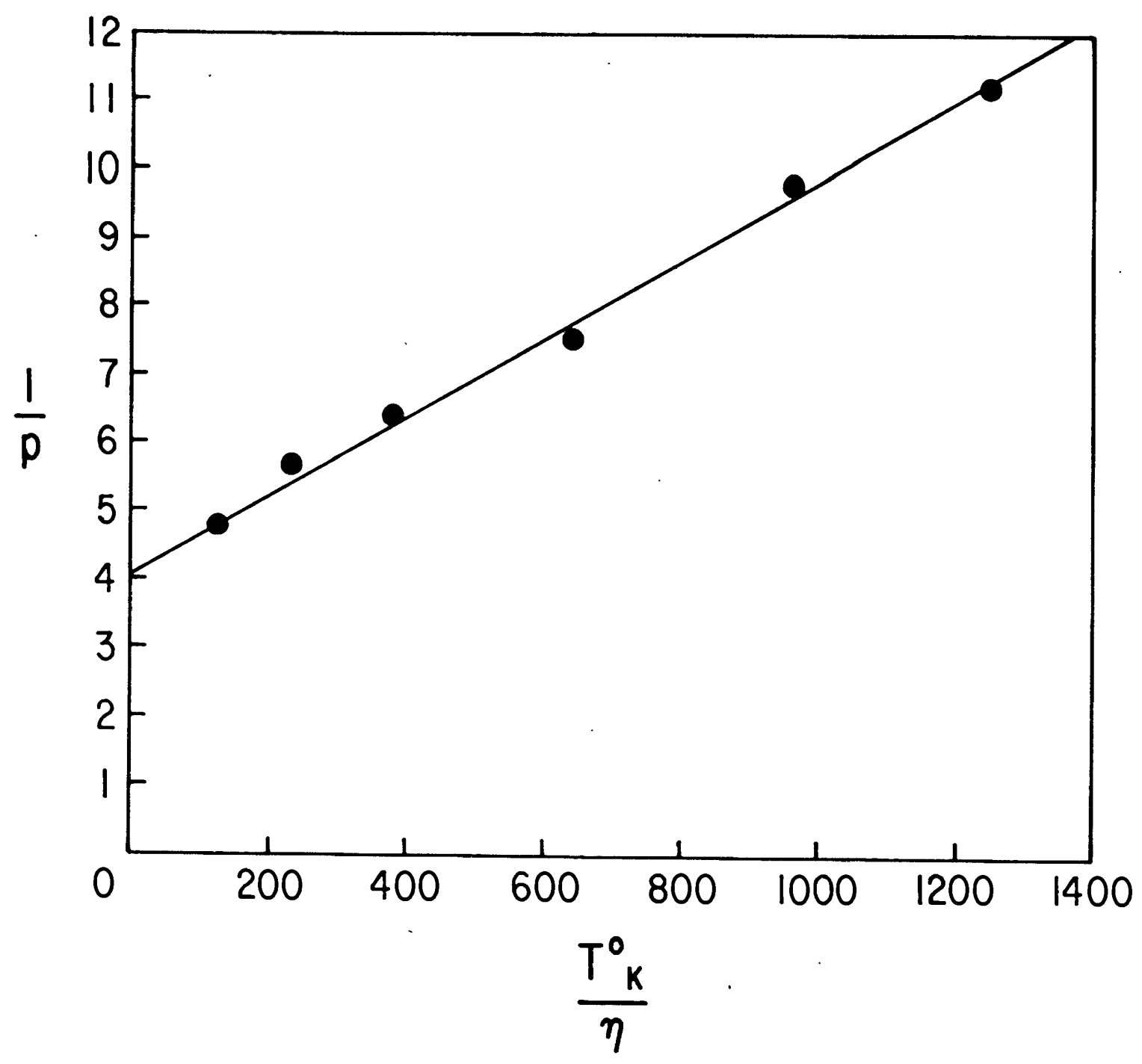

Figure 13. Plot for fluorescein $\left(10^{-6} \mathrm{M}\right)$ in various concentrations of glycerol at $25.5^{\circ} \mathrm{C}$. $\mathrm{T} / \mathrm{h}$ is in ${ }^{\circ} \mathrm{K}$ per poise. 


\begin{tabular}{|c|c|c|c|c|c|c|c|c|c|c|}
\hline \% GLYCEROL & $P_{1}$ & $P_{2}$ & $P_{3}$ & $P_{4}$ & $P_{5}$ & $\bar{x}$ & $s$ & $1 / \bar{x}$ & $n$ & $T_{K / n}^{0}$ \\
\hline $67 \%$ & .0909 & .0909 & .0833 & .0957 & .0826 & .0887 & .0056 & 11.2740 & .2390 & 1249.62 \\
\hline $70 \%$ & .1053 & .1071 & .0909 & .1008 & .1057 & .1020 & .0066 & 9.8039 & .3096 & 964.66 \\
\hline $75 \%$ & .1321 & .1346 & .1287 & .1333 & 1304 & .1312 & .0023 & 7.5873 & .4700 & 635.45 \\
\hline $80 \%$ & .1624 & .1453 & .1607 & .14 .67 & .1473 & .1565 & .0096 & 6.3898 & .7859 & 379.54 \\
\hline $85 \%$ & .1736 & .1667 & .1826 & .1765 & .1729 & .1745 & .0050 & 5.7307 & 1.2950 & 230.63 \\
\hline $90 \%$ & .2069 & .2069 & .2182 & .200 & .2097 & .2083 & .0066 & 4.8008 & 2.4143 & 123.68 \\
\hline
\end{tabular}

$T=25.5^{\circ} \mathrm{C} ; \quad\left(T_{K}=273.16+25.5=298.60\right)$

Figure 14. Table of polarization responses for fluorescein $\left(10^{-6} \mathrm{M}\right)$ in glycerol concentrations ranging from $67 \%$ to $90 \%$. Five mus were made and the average and standard deviation calculated. The reciprocal of the average for each run was determined as $1 / p$ for the ordinate values. $n$ was calculated for each glycerol concentration and $T_{K / n}^{O}$ was calculated for the abscissa values.

\section{CONCLUSIONS}

The results show that the system has good linearity for fluorescence response and polarization measurement. It is, however, limited in its sensitivity, not by the lack of photomultiplier sensitivity, but by the high light "noise" level. Considering the remaining three decades of photomultiplier sensitivity and an additional 500 volts which could be applied to the photomultiplier dynodes it is quite likely that the system sensitivity can be increased from $10^{-8} \mathrm{M}$ to $10^{-11} \mathrm{M}$ or even $10^{-12} \mathrm{M}$ if the ambient light level can be reduced sufficiently.

The use of the ellipsoidal mirror does seem to compensate for any loss in sensitivity arising from the lack of collimating optics and the relatively low-power excitation source. However, this axial optical system involving the mirror with the sample cell at the focal point may be responsible for the high ambient light level. Reflections from the front surface of the sample cell are also directed by 
the mirror to the photomultiplier. However, the axial optical system offers as compensation compactness and simplicity.

The artifically low values of $(p)$ arising from horizontal polarization of the excitation source can be corrected by introducing a depolarizer in series with the light source. This can be either a short length of non-coherent fiber optics or a series of glass plates stacked until depolarization has been accomplished. In either case the optical characteristics of the depolarizer should assure passage of light wavelengths weli into the UV region.

Although at the fluorescein concentrations and sensitivities studied, temperature fluctuations of a few degrees centigrade did not seem to effect the data, it is certain that at higher sensitivities effects would be seen. At such time it is paramount that the sample cell be temperature stabilized to within 0.1 or 0.2 degrees $C$. The results of this study are encouraging. It appears that with further work to improve the ambient light problem and provide temperature regulation of the sample cell, adequate sensitivity and stability can be achieved to satisfy the needs of a laboratory instrument. The basic design goals of compactness and simplicity have already been achieved. The addition of a microprocessor to read and interpret the signal will further enhance this device and it is believed that at that time it will be a marketable instrument. 


\section{BIBLIOGRAPHY}

1. Perrin, F., J. Phys. Radium, 7,390 (1926)

2. Weber, G., Biochem. J., 51,145 (1952)

3. Laurence, D.J.R., Biochem. J., 51,168 (1952)

4. Weber, G., J. Optical Society of America, 46,962 (1956)

5. Johnson, P. and Richards, E.G., Arch. of Biochem. and Biophy., $97,250(1962)$

6. McKay, R.H., Arch. of Biochem. and Biophy., 135,218 (1969)

7. Rosen, C., Acta Chem, Scand., 24,1849 (1970)

8. Lavore1, J. and Vernotte, C., Biochimie, 54,161 (1972)

9. Kelly, R.J. and Dandliker, W.B., Analytical Chemistry, 48,846 (1976)

10. Watson, R.A.A., Landon, J., Shaw, E.J., and Smith, D.S., Clinica Chimica Acta, 73,51 (1976)

11. Gaviola, E.Z., Z. Physik., 42,852 (1927)

12. Dushinsky, F., Acta Physiochem., 7,551 (1937)

13. Szymanowski, W., Z. Physik., 95,456 (1935)

14. Strong, J., Concepts of Classical Optics, W.H. Freeman \& Co., San Francisco, 1958, P 114-116

15. Jenkins, F.A. and White, E.H., Fundamentals of Optics, McGraw-Hi11, New York, 1957, P 513-522 